\title{
Biomasa y carbono en Guadua angustifolia y Bambusa oldhamii en dos comunidades de la sierra Nororiental de Puebla, México
}

\author{
Juan Francisco Aguirre-Cadena ${ }^{1}$, Benito Ramírez-Valverde ${ }^{1 *}$, Jorge Cadena-Iñiguez ${ }^{2}$, \\ José Pedro Juárez-Sánchez ${ }^{1}$, Laura Caso-Barrera ${ }^{1}$ \& Daniel Martínez-Carrera ${ }^{1}$ \\ 1. Colegio de Postgraduados, Campus Puebla, Km.125.5 Carretera Federal México Puebla, Santiago Momoxpan, San \\ Andrés Cholula, Puebla, México; aguirre.juanf@gmail.com, bramirez@colpos.mx, pjuarez@colpos.mx, \\ lauracaso2004@yahoo.com,dcarrera@colpos.mx \\ 2. Colegio de Postgraduados Campus San Luis Potosí, Iturbide No. 73, Salinas de Hidalgo, San Luis Potosí, México; \\ jocadena@colpos.mx \\ * Correspondencia
}

Recibido 21-V-2018. Corregido 16-VIII-2018. Aceptado 04-X-2018.

\begin{abstract}
Biomass and carbon of Guadua angustifolia and Bambusa oldhamii in two communities of the Nororiental Sierra of Puebla, México. The increase of $\mathrm{CO}_{2}$ in the atmosphere can be reduced by capturing them through the photosynthetic process, with special emphasis on fast growing perennial species such as bamboo. The objective of the present study was to quantitatively evaluate the aerial biomass and estimate the carbon content for the species Guadua angustifolia Kunth and Bambusa oldhamii Munro, in commercial plantations established in communities of the Northeast Sierra of Puebla, Mexico. The estimation of population and aerial biomass for both species is tended by a selection of $10 \%$ of plants in one hectare. The samplings were carried out from June 2016 to September 2017. For the plantation of B. Oldhamii Munro, the number of culms per plant gradually increased towards the most recent generations, from 3.6 stems in the most adult cohort to 4.5 in the youngest. The diameter of the stems and their average height show a gradual increase from the older cohorts to the recent cohorts. The total aerial biomass estimated in the plantation of B. oldhamii Munro was 103403 48 Mgha- 1 distributed in the asses of the four years. The total aerial biomass estimated in the plantation of $G$. angustifolia Kunth is 4766511 Mgha-1.
\end{abstract}

Key words: culms; diameter; carbon fraction; cohort; peasant.

Aguirre-Cadena, J. F., Ramírez-Valverde, B., Cadena-Iñiguez, J., Juárez-Sánchez, J. P., CasoBarrera, L., \& Martínez-Carrera, D. (2018). Biomasa y carbono en Guadua angustifolia y Bambusa oldhamii en dos comunidades de la sierra Nororiental de Puebla, México. Revista de Biología Tropical, 66(4), 1701-1708.

Existen evidencias que el efecto invernadero es producto del incremento de las emisiones de gases generadas por el hombre (IPCC, 2000), el $\mathrm{CO}_{2}$ ha incrementado de 280 a 375 mg. $\mathrm{kg}^{-1}$ en la atmósfera en los últimos 200 años y continúa el aumento a una tasa anual superior a $1.5 \mathrm{mg} \cdot \mathrm{kg}^{-1}$ (White, Velarde, Alegre, \& Tomich, 2005), especialmente por actividades antrópicas en combinación con eventos naturales que inciden sobre la disminución de la vegetación. También al disminuir la vegetación también se merma su capacidad para incorporar el carbono al proceso fotosintético en los diferentes órganos de las plantas. La fotosíntesis es una estrategia para disminuir el $\mathrm{CO}_{2}$ en el ambiente, con especial énfasis en algunas especies perennes de amplia distribución y rápido crecimiento, como: Dendrocalamus latiorus (Munro) var. latiorus (Lin), Guadua angustifolia Kunth, $G$ aculeata (Rupr. ex E. Fourn) 
y Phyllostachys heterocycla var. pubescens (J. Houz) (Lobovikov, Paudel, Piazza, Ren, $\& \mathrm{Wu}, 2007)$.

Algunas especies de bambú tienen altas tasas de crecimiento de 7.5 a $100 \mathrm{~cm}$ por día (Buckingham et al., 2011) que favorecen la acumulación de carbono orgánico (Lobovikov, Schoene, \& Lou, 2012) en cantidades sustanciales para mitigar los efectos del cambio climático (Nath, Lal, \& Das, 2015). Se ha demostrado con $P$. pubescens en China donde presenta diferente capacidad de fijación de carbono con $31 \mathrm{Mg} \cdot \mathrm{ha}^{-1}$ (Yiping, Yanxia, Breckinghan, Henley \& Guomo, 2011), $37 \mathrm{Mgha}^{-1}$ (Huaqiang et al., 2011) a $40 \mathrm{Mgha}^{-1}$ (Zhang, et al., 2014) al año. En Taiwán con $P$. heterocycla se cita fijación anual de $41 \mathrm{Mg}^{-h a^{-1}} \mathrm{y}$ con $P$. makinoi Ohwi de 50 Mg.ha-1 (Yen \& Lee, 2011). En India con D. strictus (Roxb.) Nees se

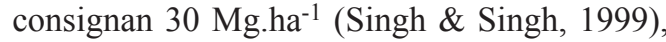
con Pseudostachyum polymorphum Munro 23 Mg.ha-1 y con Schizostachyum dulloa (Gam-

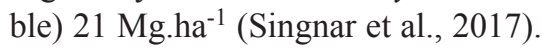

Bambusa oldhamii Munro fija 51.98

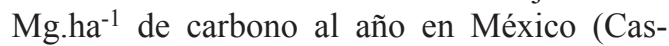
tañeda, Vargas, Gómez, Valdez, \& Vaquera, 2005), en Bolivia 27.53 Mg.ha ${ }^{-1}$ (Rojas-Quiroga et al., 2013) y en Costa Rica $46.91{\mathrm{Mg} . \mathrm{ha}^{-1}}^{-1}$ (Elizondo-Briceño et al., 2016). En Colombia

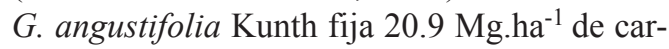
bono (Camargo et al., 2010), mientras que otros autores citan amplia escala de fijación de 24.6

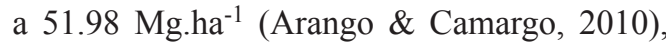

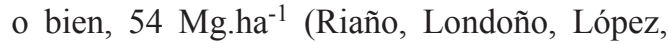
\& Gómez, 2002). Con base en lo anterior, se evaluó cuantitativamente la biomasa aérea de G. angustifolia y B. oldhamii, para determinar la tasa de secuestro de carbono y caracterizar los principales rasgos de manejo de bambú en comunidades de los municipios de Teziutlán y Hueytamalco, Puebla, México.

\section{MATERIALES Y MÉTODOS}

La investigación se desarrolló con dos especies y dos localidades. La primera con $B$. oldhamii en el Rancho La soledad del municipio de Teziutlán, Puebla, México (1946’
- 195' N \& 97²19' - 97²5' W) a 1600 m de altitud con clima templado húmedo, precipitación promedio de $2350 \mathrm{~mm}$ anuales, temperatura media de $18{ }^{\circ} \mathrm{C}$ y suelos Andosoles. G. angustifolia, se estudió en el Rancho El bambusal, comunidad Loma Alta, en Hueyta-

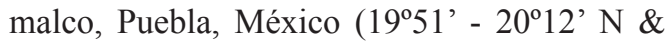
$\left.97^{\circ} 12^{\prime}-97^{\circ} 23^{\prime} \mathrm{W}\right)$ a $582 \mathrm{~m}$ de altitud. El clima es semicálido húmedo con lluvias en verano, la precipitación promedio anual es de $2000 \mathrm{~mm}$ y la temperatura media de $22{ }^{\circ} \mathrm{C}$ con suelos regosoles (INEGI, 2010). Las especies presentan patrones de crecimiento diferentes en el rizoma. En G. angustifolia Kunth es monopodial y en B. oldhamii Munro es simpodial. Estos atributos fueron estudiados con metodologías distintas en cada especie.

Tamaño de muestra y variables: En ambas especies, la estimación de población y distribución de biomasa aérea se obtuvo a una selección del $10 \%$ de una densidad de 400 plantas en una hectárea (ha), a las cuales se registró diámetro de tallo $(\mathrm{cm})$ a $1.3 \mathrm{~m}$ de altura; tomando en cuenta el establecimiento del cultivo el año 2011. Los muestreos comenzaron el año 2016 cuando la planta ya tenía una edad comercial de 5 años, considerando el año 2016 como año uno para los diámetros comerciales de los culmos. La altura de las plantas se determinó mediante el equipo Field Map Data Collector (IFER-Monitoring and Mapping Solutions, Ltd, Republica Checa).

La estimación de la densidad de $B$. oldhamii Munro en la plantación establecida en 2011 se obtuvo con los datos de 40 individuos con follaje maduro tomados al azar en los culmos como indicador de máximo crecimiento. En $G$. angustifolia Kunth se determinó en una muestra de 400 plantas y se consideró el número de culmos que emergieron en el año 1 (2016). A través de la relación del diámetro y el año de la planta se calculó la biomasa total de ambas especies para estimar el coeficiente de regresión por el modelo $Y=\alpha \mathrm{D} \beta$ (Castañeda et al., 2005).

Para ambas especies se aplicó el ajuste propuesto por Baskerville (1972) para eliminar el sesgo asociado a la transformación 
logarítmica del modelo, que expresado nuevamente en escala aritmética se define como:

$$
\mathrm{Yci}=\mathrm{e}(\alpha+\beta \ln (\mathrm{D})+\mathrm{CME} / 2)
$$

(Wiant \& Harner,1979)

Donde Yci es la biomasa calculada del iésimo componente, e es la base del logaritmo natural y CME es el cuadrado medio del error calculado por componente y edad.

Los parámetros usados para obtener biomasa en B. oldhamii Munro fueron constantes, pendientes y valores de $\mathrm{R}^{2}$ para las ecuaciones de biomasa total. En G. angustifolia Kunth se utilizaron parámetros que relacionaron la biomasa seca de tallos, ramas y follaje (Cuadro 1).

\section{RESULTADOS}

La distribución de los culmos en el terreno fue diferente por especie (e.g. G. angustifolia y $B$. oldhamii) debido a sus diferencias en morfología y crecimiento (Fig. 1). La densidad de culmos en G. angustifolia fue de 3300 $\mathrm{ha}^{-1}$, la cual y ésta densidad de población que representa un valor intermedio en plantaciones comerciales establecidas.
La biomasa en brotes con el mismo diámetro se incrementó Se incrementó la biomasa en brotes con el mismo diámetro en $B$. oldhamii en comparación a G. angustifolia. Además, se presentó relación de crecimiento definido por edades y diámetro en las dos especies. En el primer año, los culmos estuvieron definidos por edades más tempranas en G. angustifolia hasta alcanzar la madurez final, es decir, el diámetro no cambió con el tiempo.

El crecimiento simpoidal de B. oldhamii aumentó su dinámica de crecimiento y en consecuencia la densidad de plantas a través del tiempo. Después de 2 años se incrementó $24 \%$ el número de culmos. Sin embargo, en el año tres, el aumento representó $110 \%$ con relación al año anterior, y en el cuarto año, disminuyó la emisión de tallos a un nivel inferior a la población inicial (Fig. 2). La extracción de culmos abre espacio para el desarrollo de nuevos brotes y se refleja en aumento de sus dimensiones, también se incrementa el intervalo dimétrico de la especie hasta el año 1 , con diámetros de 10 $\mathrm{cm}$. En especies de bambú de tipo simpodial, los culmos jóvenes son más productivos que los viejos, por lo que esta condición genera la recomendación de no cosechar los culmos menores de cuatro años de edad.

CUADRO 1

Constantes, parámetros y valores de $\mathrm{R}^{2}$ para las ecuaciones de biomasa aérea de B. oldhamii y G. angustifolia

TABLE 1

Constants, parameters and values of $\mathrm{R}^{2}$ for the aerial biomass equations of B. oldhamii and G. angustifolia

\begin{tabular}{ccccc}
\hline \multicolumn{5}{c}{ Coeficientes usados para B. oldhamii* } \\
Edad (años) & Alfa & Beta & CME & $\mathrm{R}^{2}$ \\
1 & 6.85 & 1.24 & 0.0142 & 0.77 \\
2 & 5.75 & 1.84 & 0.0112 & 0.79 \\
3 & 5.07 & 2.23 & 0.0144 & 0.95 \\
4 & 6.02 & 1.64 & 0.0153 & 0.87 \\
Estructura de la planta & Parámetros y valores para G. angustifolia** & \\
Tallos & Alfa & Beta & CME & $\mathrm{R}^{2}$ \\
Ramas & 2.6896 & 0.961 & 0.0478 & 0.9524 \\
Follaje & 0.0510 & 1.443 & 0.1214 & 0.8762 \\
Total & $9.4 \times 10^{-6}$ & 4.027 & 0.3665 & 0.7153 \\
\hline
\end{tabular}

*(Castañeda et al., 2005); **(Riaño et al., 2002). 

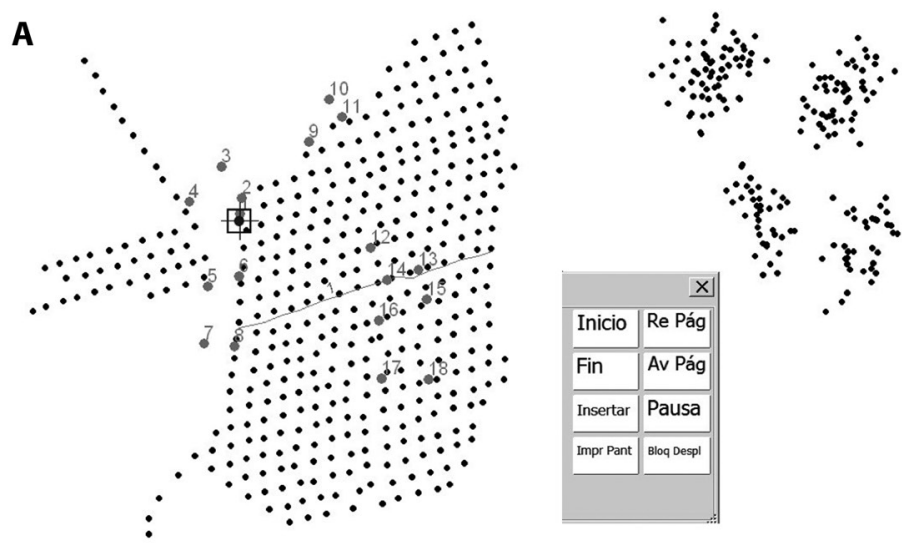

Fig. 1. (A) Distribución de los culmos de B. oldhamii, en Rancho la Soledad, y G. angustifolia (B) Rancho el Bambusal, Puebla, México.

Fig. 1. (A) Distribution of the culms of B. oldhamii, in Rancho la Soledad, and G. angustifolia. (B) Rancho el Bambusal, Puebla, Mexico.

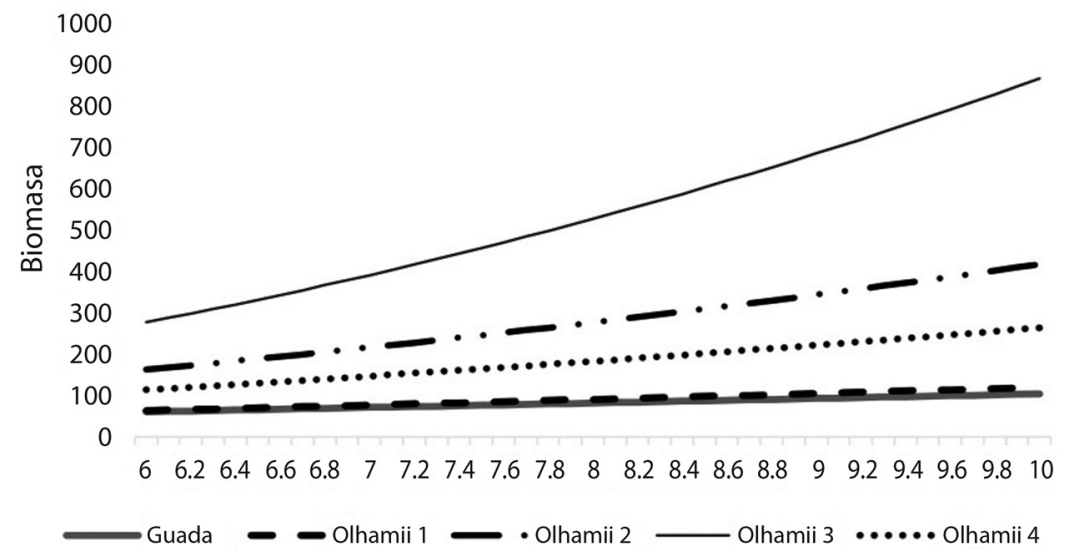

Fig. 2. Relación del diámetro y biomasa de G. angustifolia y B. oldhamii en plantaciones forestales de Puebla, México.

Fig. 2. Relation of the diameter and biomass of G. angustifolia and B. oldhamii in forest plantations of Puebla, Mexico.

Disminuyeron los culmos en B. oldhamii después del año cuatro de siembra a este tiempo, la relación diámetro-biomasa está por debajo de la curva de crecimiento de los años anteriores. En cambio, las plantas jóvenes incrementan el desarrollo de ramas laterales. $B$. oldhamii produce más biomasa en comparación a G. Angustifolia y B. oldhamii incrementa su crecimiento dimétrico culmo por culmo.

El número de culmos por planta aumentó gradualmente hacia las generaciones más recientes, pasó de 3.96 en la cohorte más adulta (cuatro años de edad) a 4.5 en la más joven (un año de edad) (Cuadro 2). El crecimiento del diámetro en los culmos de $B$. oldhamii fue exponencial durante los tres primeros años después de la siembra (Fig. 3 a), sin embargo, a este tiempo, el crecimiento de la población no alcanza la estabilidad (Fig. 3 b), el culmo continúa creciendo y es característico en plantaciones jóvenes.

La biomasa aérea total estimada de $B$. oldhammi con base a la ecuación de Castañeda et al., (2005) fue de $10340348 \mathrm{Mgha}^{-1}$ distribuida 
CUADRO 2

Promedios de los culmos de B. oldhamii en cuatro cohortes en Teziutlán Puebla, México

TABLE 2

Averages of the culms of $B$. oldhamii in four cohorts in Teziutlán Puebla, México

\begin{tabular}{lcccc}
\multicolumn{4}{c}{ Variable } & \multicolumn{4}{c}{ Edad de culmo (años) } \\
& $4(2013)$ & $3(2014)$ & $2(2015)$ & $1(2016)$ \\
Culmos planta $^{-1}$ & 3.96 & 12.05 & 5.71 & 4.57 \\
Culmos ha $^{-1}$ & 120 & 470 & 223 & 184 \\
${\text { Diámetro }\left(\mathrm{cm} \mathrm{planta}^{-1}\right)}^{\text {Intervalo diamétrico }}$ & 4.96 & 6.3 & 7.49 & 7.33 \\
Altura (m planta & $(1.7-6.6)$ & $(4.1-8.9)$ & $(4.3-9.7)$ & $(2.6-10)$ \\
Intervalo en altura & 9.06 & 14.65 & 18.05 & 17.4 \\
& $(7.4-4.6)$ & $(12.3-8.8)$ & $(14.8-21.5)$ & $(13.9-0.45)$
\end{tabular}
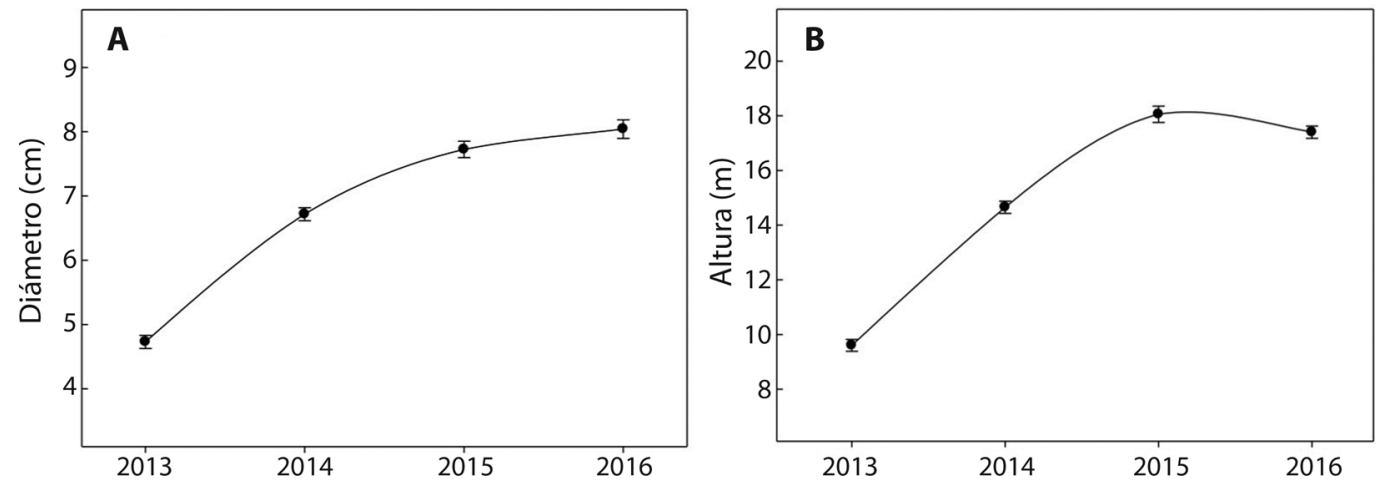

Fig. 3. Diámetro y altura de los culmos de Bambusa oldhamii en los últimos cuatro años, en plantaciones ubicadas en Teziutlán Puebla, México.

Fig. 3. Diameter and height of the culms of Bambusa oldhamii in the last four years, in plantations located in Teziutlán Puebla, México.

en culmos de los cuatro años. Lo anterior representó acumulación promedio de $25 \mathrm{Mgha}^{-1}$ por año. La biomasa aérea total estimada con base en la ecuación de Riaño et al., (2002) en la plantación de G. Angustifolia fue de 47665 $11 \mathrm{Mgha}^{-1}$. Para ambos casos y de acuerdo con Brown (1997), quien cita que la biomasa total acumula $50 \%$ del carbono en tejido vegetal, se estimó, que la cantidad de carbono retenida y calculada en la parte aérea de la plantación de B. oldhammi sería de $12.5 \mathrm{Mgha}^{-1}$, y para $G$. Angustifolia de $23.8 \mathrm{Mgha}^{-1}$ por año.

\section{DISCUSIÓN}

La densidad de culmos en G. angustifolia representa un valor intermedio en otras plantaciones establecidas, con valores de 1000

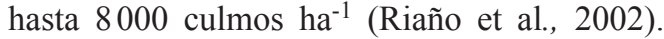
Sin embargo, la densidad cambia con el tiempo al incorporarse más culmos cada año (Riaño et al., 2002), como sucede con B. oldhamii por su desarrollo simpodial (Castañeda et al., 2005). Las diferencias encontradas en los niveles de población, sugieren un proceso de regulación natural por parte de la planta a través del tiempo.

La disminución de culmos en $B$. oldhamii después del año cuatro de siembra es probable que se deba a la competencia por espacio lateral en las plantaciones y la concomitante disminución de la actividad fotosintética. A este tiempo, la relación diámetro-biomasa está por debajo de la curva de crecimiento de los años 
anteriores. No obstante, las plantas jóvenes incrementan el desarrollo de ramas laterales y la distribución de la biomasa está influenciada por la disponibilidad de la luz y la edad de las especies (Anten \& Hirose, 1998). B. oldhamii produce más biomasa en comparación a $G$. Angustifolia y B. oldhamii incrementa su crecimiento dimétrico. El efecto anterior sugiere, mayor fijación de carbono e incremento de biomasa aérea y radical (Fig. 2).

El crecimiento diferencial entre altura y diámetro del culmo en las dos especies estuvo definido por la edad. En el primer año en $G$. angustifolia el diámetro no varió y este comportamiento se atribuye a la ausencia de crecimiento secundario (Judziewicz, 1999). Resultados semejantes a los encontrados en esta investigación cita Cusack (1997) con alturas de $18 \mathrm{~m}$ y diámetros de $10 \mathrm{~cm}$ en la misma especie. En cambio, en B. oldhamii se incrementó de manera exponencial durante los tres primeros años. Este crecimiento es característico de plantaciones jóvenes (Chrystanty, Mailly, \& Kimmins, 1996), pero con altura inferior entre 6 y $9 \mathrm{~m}$ y diámetros de 5 a $6 \mathrm{~cm}$ en los culmos de B. oldhamii (Shilin, Naixun, $\&$ Maoyi, 1994). Es probable que el incremento contrastante en crecimiento en la misma especie esté influenciado en condiciones ambientales en cada región.

La cantidad de biomasa aérea y los valores de Carbono almacenado calculados en la parte aérea para ambas especies está dentro de las cantidades registradas otros autores (Kleinhenz \& Midmore, 2001; Arango \& Camargo, 2010) con variaciones locales influenciadas por condiciones bióticas y abióticas, edad y densidad de la población. El rápido crecimiento de las dos especies y la aceptación local en la Sierra Nor Oriental de Puebla permite considerarlas como sumidero de carbono, además, aumenta las posibilidades de usos y aplicaciones de la planta.

En el presente trabajo se demuestra que las plantaciones comerciales de bambú en la Sierra Nor Oriental de Puebla, que utilizan especies como G. Angustifolia y B. oldhamii por su facilidad de manejo y tradición. El crecimiento simpodial de B. Oldhamii permite pronosticar su crecimiento con facilidad. Tomando en cuenta que $B$. Oldhamii es una especie introducida en la zona hace más de 15 años, ha desarrollado un papel importante en el desarrollo económico de la zona por su facilidad de manejo y capacidad de almacenar carbono; estas especies pretenden ser el potencial económico de la zona mediante las distintas formas de aprovechamiento del bambú.

Con los resultados obtenidos de la biomasa aérea total estimada en la plantación de $B$. oldhamii de $10340348 \mathrm{Mgha}^{-1}$ distribuida en los culmos de los cuatro años y los concomitantes beneficios potenciales al ambiente a medida que aumenta su diámetro, presenta diferencia notable en comparación con G. Angustifolia que logra $50 \%$ menos biomasa aérea total estimada. Lo anterior permite proponer a $B$. oldhamii en la zona como especie promisoria de bambú para disminuir las concentraciones de carbono.

Declaración de ética: los autores declaran que todos están de acuerdo con esta publicación y que han hecho aportes que justifican su autoría; que no hay conflicto de interés de cualquier tipo; y que han cumplido con todos los requisitos y procedimientos éticos y legales pertinentes. El documento firmado se encuentra en los archivos de la revista.

\section{RESUMEN}

El incremento de $\mathrm{CO}_{2}$ en la atmósfera puede disminuir mediante su captura por plantas a través del proceso fotosintético, con especial énfasis en especies perennes de rápido crecimiento como los bambús. El objetivo fue evaluar cuantitativamente la biomasa aérea y estimar el contenido de carbono para las especies Guadua angustifolia y Bambusa oldhamii, en plantaciones comerciales establecidas en dos comunidades de la Sierra Nororiental de Puebla, México. La estimación de población y biomasa aérea para ambas especies se obtuvo mediante una selección del 10\% de plantas en una hectárea. Los muestreos se realizaron de junio 2016 a septiembre 2017. Para la plantación de B. oldhamii, el número de culmos por planta aumentó gradualmente hacia las generaciones más recientes, de 3.6 tallos en la cohorte más adulta a 4.5 en la más joven. El diámetro de los tallos y su altura promedio presenta aumento gradual de las cohortes de mayor edad a las más recientes. La biomasa 
aérea total estimada en la plantación de $B$. oldhamii fue de 10340348 Mg.ha- ${ }^{1}$ distribuida en los culmos de los cuatro años. La biomasa aérea total estimada en la plantación de G. angustifolia es $4766511 \mathrm{Mg} \cdot \mathrm{ha}^{-1}$.

Palabras clave: culmos; diámetro; fracción de carbono; cohorte; campesino.

\section{REFERENCIAS}

Anten, N. P., \& Hirose, T. (1998). Biomass allocation and light partitioning among dominant and subordinate individuals in Xanthium canadense stands. Annals of Botany, 82, 665-673.

Arango, A., \& Camargo, J. C. (2010). Bosques de guadua del Eje Cafetero de Colombia: oportunidades para su inclusión en los mercados de carbono y en el programa redd+. Recursos Naturales y Ambiente, 61, 77-85. DOI: 10.13140/RG.2.1.3491.0569

Baskerville, G. L. (1972). Use of logarithmic regression in the estimation of plant biomass, Canadian Journal of Forest Research, 2, 49-53. DOI: 10.1139/x72-009

Brown, S. (1997). Estimating biomass and biomass change of tropical forests: a primer. FAO Forest Paper 143. Roma: FAO. Recuperado de: http://www.fao.org/ docrep/w4095e/w4095e00.htm

Buckingham, K., Jepson, P., Wu, L., Ramanuja Rao, I. V., Jiang, S., Liese, W., Lou, Y., \& Fu, M. (2011). The potential of bamboo is constrained by outmoded policy frames. Ambio, 40, 544-548. DOI: 10.1007/ s13280-011-0138-4

Camargo, J., Chará, J., Giraldo, L. P., Chará, M., \& Ximena, G. (2010). Beneficios de los corredores ribereños de Guadua angustifolia en la protección de ambientes acuáticos en la Ecorregión cafetera de Colombia. Efectos sobre las propiedades del suelo. Recursos Naturales y Ambiente, 61, 53-59. DOI: 10.13140/ RG.2.1.3491.0569

Castañeda, A., Vargas, J., Gómez, A., Valdez, J., \& Vaquera, H. (2005). Acumulación de carbono en la biomasa aérea en una plantación de Bambusa oldhamii. Agrociencia, 30(1), 107-113.

Chrystanty, L., Mailly, D., \& Kimmins, J. P. (1996). Without bamboo, the land dies: Biomasss, literfall, and soil organic matter dynamics of a Javanese bamboo talunkebun system. Forest Ecology Management, 87, 75-88. DOI: 10.1016/S0378-1127(96)03834-0

Cusack, V. (1997). Bamboo Rediscovered. Trentham, Victoria: Earth Garden Books. Recuperado de http://trove. nla.gov.au/work/24676120?q\&versionId=45621233

Elizondo-Briceño, E., Barquero, V., Fonseca, V. L., Bonilla, G. M., \& Segura, E. E. (2016). Potencial de crecimiento y almacenamiento de carbono en plantaciones de bambú Guadua (Guadua angustifolia) en la Zona Sur de Costa Rica. Primer congreso de bambú, México. Recuperado de https://1 ercongresobambu.files. wordpress.com/2017/02/elemer-bricec3b1o-potencial-de-crecimiento-y-almacenamiento-de-carbonoen-plantaciones-de-bambc3ba-guadua-en-la-zonasur-de-costa-rica.pdf

Huaqiang, D., Guomo, Z., Hongli, G., Wenyi, F., Xiaojun, X., Weiliang, F., \& Yongjun, S. (2011). Satellitebased carbon stock estimation for bamboo forest with a non-linear partial least square regression technique. International Journal of Remote Sensing, 33(6), 1917-1933. DOI: 10.1080/01431161.2011.603379

INEGI. (2010). Marco Geoestadístico 2010 (Versión 4.3). Teziutlán, Puebla: Compendio de información geográfica municipal de los Estados Unidos Mexicanos. Recuperado de http://www.inegi.org.mx/geo/contenidos/topografia/compendio.aspx

IPCC (Intergovernmental Panel on Climate Change). (2000). Land use, Land-use Change and Forestry. Cambridge, U. K.: Cambridge University, Press. Recuperado de http://www.ipcc.ch/ipccreports/sres/ land_use/index.php?idp $=0$

Judziewicz, E. J., Clark, L. G., Londoño, X., \& Stern, J. M. (1999). American Bamboos. Washington, D. C.: Smithsonian Institution Press.

Kleinhenz, V., \& Midmore, D. J. (2001). Aspects of bamboo agronomy. Advances in Agronomy, 74, 99-149.

Lobovikov, M., Paudel, S., Piazza, M., Ren, H., \& Wu, J. (2007). World Bamboo Resources. Rome, Italy: FAO. Recuperado de http://www.fao.org/docrep/010/ a1243e/a1243e00.htm

Lobovikov, M., Schoene, D., \& Lou, Y. (2012). Bamboo in climate change and rural livelihoods. Mitigation and Adaptation Strategies for Global Change, 17(3), 261276. DOI: $10.1007 / \mathrm{s} 11027-011-9324-8$

Nath, A. J., Lal, R., \& Das, A. K. (2015). Managing woody bamboos for carbon farming and carbon trading. Global Ecology and Conservation, 3, 654-663. DOI: 10.1016/j.gecco.2015.03.002

Riaño, N. M., Londoño, X., López, Y., \& Gómez, J. H. (2002). Plant growth and biomass distribution on Guadua angustifolia Kunth in relation to ageing in Valle del Cauca-Colombia. Bamboo Science and Culture: The Journal of the American Bamboo Society, 16(1), 43-51.

Rojas-Quiroga, R., Li, T., Lora, G., \& Andersen, L. (2013). A measurement of the carbon sequestration potential of Guadua angustifolia in the Carrasco National Park, Bolivia (Development Research Working Paper series, no. 04/2013). La Paz, Bolivia: Institute for Advanced Development Studies. Recuperado de http://www.inesad.edu.bo/pdf/wp2013/wp04_2013. pdf 
Shilin, Z., Naixun, M., \& Maoyi, F. (1994). A Compendium of Chinese Bamboo. Nanjing, China: China Forestry Publishing House.

Singh, A. N., \& Singh, J. S. (1999). Biomass, net primary production and impact of bamboo plantation on soil redevelopment in a dry tropical region. Forest Ecology and Management, 119, 195-207. DOI: 10.1016/ S0378-1127(98)00523-4

Singnar, P., Das, C. M., Sileshi, W. G., Brahma, B., Nath, J, A., \& Das, K. A. (2017). Allometric scaling, biomass accumulation and carbon stocks in different aged stands of thin-walled bamboos Schizostachyum dullooa, Pseudostachyum polymorphum and Melocanna baccifera. Forest Ecology and Management, 395, 81-91. DOI: 10.1016/S0378-1127(17)30599-6

White, D., Velarde, S. J., Alegre, J. C., \& Tomich, T. P. (2005). Alternatives to Slash-and-Burn (ASB) in Peru, Summary Report and Synthesis of Phase II. Alternatives to Slash-and-Burn Programme, Nairobi, Kenya. Kenya: Consultative Group on International Agricultural Research. Recuperado de http://www. asb.cgiar.org
Wiant, H. V. \& Harner, E. J. (1979). Percent bias and standard error in logarithmic regression. Forest Science, 25(1), 167-168.

Yen, T. M., \& Lee, J. S. (2011). Comparing aboveground carbon sequestration between moso bamboo (Phyllostachys heterocycla) and China fir (Cunning hamialanceolata) forests based on the allometric model. Forest Ecology and Management, 261, 9951002. DOI: $10.1016 /$ j.foreco.2010.12.015

Yiping, L., Yanxia, L., Breckinghan, K., Henley, G., \& Guomo, Z. (2011). Bamboo and climate change mitigation. International Network for Bamboo and Rattan (Inbar). Technical report no 32. Beijing, China: The International Network for Bamboo and Rattan. Recuperado de www.inbar.int

Zhang, H., Zhuang, S., Sun, Bo, Ji, H., Li, C., \& Zhou, S. (2014). Estimation of biomass and carbon storage of moso bamboo (Phyllostachys pubescens Mazel ex Houz.) in southern China using a diameter-age bivariate distribution model. Forestry, 87, 674-668. DOI: 10.1093/forestry/cpu028 